\title{
Microphysical properties and fall speed measurements of snow ice crystals using the Dual Ice Crystal Imager (D-ICI)
}

\author{
Thomas Kuhn and Sandra Vázquez-Martín \\ Luleå University of Technology, Department of Computer Science, Electrical and Space Engineering, \\ Division of Space Technology, 98128 Kiruna, Sweden
}

Correspondence: Thomas Kuhn (thomas.kuhn@ltu.se)

Received: 20 September 2019 - Discussion started: 4 November 2019

Revised: 4 February 2020 - Accepted: 7 February 2020 - Published: 16 March 2020

\begin{abstract}
Accurate predictions of snowfall require good knowledge of the microphysical properties of the snow ice crystals and particles. Shape is an important parameter as it strongly influences the scattering properties of the ice particles, and thus their response to remote sensing techniques such as radar measurements. The fall speed of ice particles is another important parameter for both numerical forecast models as well as representation of ice clouds and snow in climate models, as it is responsible for the rate of removal of ice from these models.

We describe a new ground-based in situ instrument, the Dual Ice Crystal Imager (D-ICI), to determine snow ice crystal properties and fall speed simultaneously. The instrument takes two high-resolution pictures of the same falling ice particle from two different viewing directions. Both cameras use a microscope-like setup resulting in an image pixel resolution of approximately $4 \mu \mathrm{m}$ pixel $^{-1}$. One viewing direction is horizontal and is used to determine fall speed by means of a double exposure. For this purpose, two bright flashes of a light-emitting diode behind the camera illuminate the falling ice particle and create this double exposure, and the vertical displacement of the particle provides its fall speed. The other viewing direction is close-to-vertical and is used to provide size and shape information from single-exposure images. This viewing geometry is chosen instead of a horizontal one because shape and size of ice particles as viewed in the vertical direction are more relevant than these properties viewed horizontally, as the vertical fall speed is more strongly influenced by the vertically viewed properties. In addition, a comparison with remote sensing instruments that mostly have a vertical or close-to-vertical viewing geometry
\end{abstract}

is favoured when the particle properties are measured in the same direction.

The instrument has been tested in Kiruna, northern Sweden $\left(67.8^{\circ} \mathrm{N}, 20.4^{\circ} \mathrm{E}\right)$. Measurements are demonstrated with images from different snow events, and the determined snow ice crystal properties are presented.

\section{Introduction}

Accurate knowledge of atmospheric ice crystals and snowflakes, or snow particles, is needed for meteorological forecast and climate models (see, e.g. Tao et al., 2003; Stoelinga et al., 2003). In particular, microphysical properties that are difficult to measure with remote sensing such as size, area, shape, and fall speed are important. Knowledge about these microphysical properties can, for instance, help improve parameterizations of snow particles in atmospheric models.

To retrieve precipitation amount reaching the ground, the microphysical properties of the snow particles on their way down have to be known. Fall velocity plays a significant role for modelling of the microphysical processes. It determines the number of particles present in the measuring volume and the snowfall rate, or the rate of particle removal from clouds.

Other important microphysical properties of snow particles are their shape and morphology, not only for investigating growth processes. Snow particle shape and morphology affect radar measurements (see, e.g. Sun et al., 2011; Matrosov et al., 2012; Marchand et al., 2013) and microwave brightness temperature (Kneifel et al., 2010), and are significant for optical remote sensing retrievals of cloud properties 
(see, e.g. Yang et al., 2008; Baum et al., 2011; Xie et al., 2011; Loeb et al., 2018).

Snowfall has long been monitored by ground-based instruments. However, instruments that can measure size, shape, and fall speed simultaneously are still scarce.

Instruments can be classified into different categories depending on what is measured primarily. Disdrometers, for example, measure shadow or attenuation as droplets or snow particles pass one or several light beams. Fall speed can be estimated either from the duration between the two beam passages, in case of instruments that have two parallel beams with known vertical spacing, or from the duration of attenuation. Three common disdrometers are Parsivel (particle size velocity disdrometer; see, e.g. Battaglia et al., 2010), 2DVD (two-dimensional video disdrometer; see, e.g. Kruger and Krajewski, 2002) and HVSD (hydrometeor velocity and shape detector; see, e.g. Barthazy et al., 2004). The latter two are optical array instruments, where the shadow of the particles is detected with a linear array of detectors. Thus, a shadow image can be reconstructed and the particle shape discerned. Disdrometers, generally, are designed for snowflakes with larger dimensions and their size limit (pixel size) is as large as $200 \mu \mathrm{m}$.

Another category of instruments uses camera systems for optical imaging of snow particles. One example is SVI (Snowflake Video Imager, Newman et al., 2009; in a newer version also called PIP, Particle Imaging Package). It consists of a video camera with a pixel resolution of $100 \mu \mathrm{m}$ and a halogen lamp which is placed approximately $2 \mathrm{~m}$ from the camera for background illumination. The higher frame rate $\left(380 \mathrm{~s}^{-1}\right)$ of PIP allows determination of the fall speed with image analysis software that follows particles over several frames. The ice crystal imaging (ICI) probe uses a highresolution charge-coupled device (CCD) camera system with a pixel resolution of $4.2 \mu \mathrm{m}$ (Kuhn and Gultepe, 2016). It has also been used to measure fall speed by double exposing snow particles using two flashes of illuminating light triggered at a known time difference.

There are instruments designed for aircraft that have also been used on the ground for snow measurements. CIP (cloud imaging probe, see Baumgardner et al., 2001) is an optical array probe and has been used on the ground as a GCIP (Gultepe et al., 2014). VIPS is a video camera system (see Appendix of McFarquhar and Heymsfield, 1996) with a high pixel resolution. On the ground, it has been used for example for ice fog particles with a pixel resolution of $1.1 \mu \mathrm{m}$ (Schmitt et al., 2013). CPI (Cloud Particle Imager, Lawson et al., 2001) uses a CCD camera to produce shadow graphs or images in cases where ice particles are in focus, with a pixel resolution of $2.3 \mu \mathrm{m}$. All three instruments used aspiration to produce similar inlet flows as encountered on the aircraft.

Holographic imaging has the advantage of a larger depth of field when compared to so-called "in-focus imaging". Shadow-like images of out-of-focus particles can be recon- structed and their position determined. Holographic Detector for Clouds (HOLODEC) is an aircraft instrument (Fugal et al., 2004) and HOLIMO (Holographic Imager) is a ground-based instrument (Amsler et al., 2009). HOLIMO II, a newer version, is used for ground-based in situ measurements of particles in mixed-phase clouds (Henneberger et al., 2013).

PHIPS (Particle Habit Imaging and Polar Scattering) uses a combination of optical imaging and scattering (with polar nephelometer). A first version of the instrument had a high pixel resolution, better than the $2 \mu \mathrm{m}$ optical resolving power (Schön et al., 2011). The next version, PHIPS-AIDA, added a second camera at an angle of $60^{\circ}$ to the first camera to produce stereo images and has been used for cloud chamber experiments (Abdelmonem et al., 2011). MASC (Multi-Angle Snowflake Camera) uses three cameras to image snow from three angles, while simultaneously measuring their fall speed with two sets of IR emitter-receiver pairs registering the shadow twice (Garrett et al., 2012). The cameras are viewing horizontally and are separated by $36^{\circ}$. Different pixel resolutions may be used by the cameras, and the version described by Garrett et al. (2012) used pixel resolutions between 9 and $32 \mu \mathrm{m}$. Such multi-imagers provide more detail about the 3-D structure of the snow particle that adds valuable information to the microphysical data collected by imaging instruments. This is useful, for example, to provide better estimates of snow particle mass.

This work presents a novel instrument that uses two cameras for simultaneous particle imaging and fall speed measurement. It is called the Dual Ice Crystal Imager (D-ICI) and is a development of ICI (Kuhn and Gultepe, 2016). The DICI has two cameras; the first camera uses a horizontal viewing direction (side view), whereas the second camera views the falling snow particle vertically (top view).

The cross-sectional area as seen from the top is better related to the particle drag and terminal fall velocity than the area determined from the side view. Additionally, particle size and area from the top view are also more useful when comparing to optical remote sensing, which often uses vertical viewing geometries too. Sections 2 and 3 describe the design of D-ICI and data processing methods; Sect. 4 presents measurements to evaluate the instrument's capabilities.

\section{Instrument}

\subsection{Instrument setup}

D-ICI uses passive sampling with a vertically pointing inlet. Its setup can be seen schematically in Fig. 1 .

Ice particles, i.e. small ice crystals, snow crystals, and snowflakes, falling into the inlet will fall down the sampling tube and traverse the optical cell. In the centre of the optical cell is the sensing volume. If a particle is falling through the sensing volume, it is detected by the detecting optics. Upon 

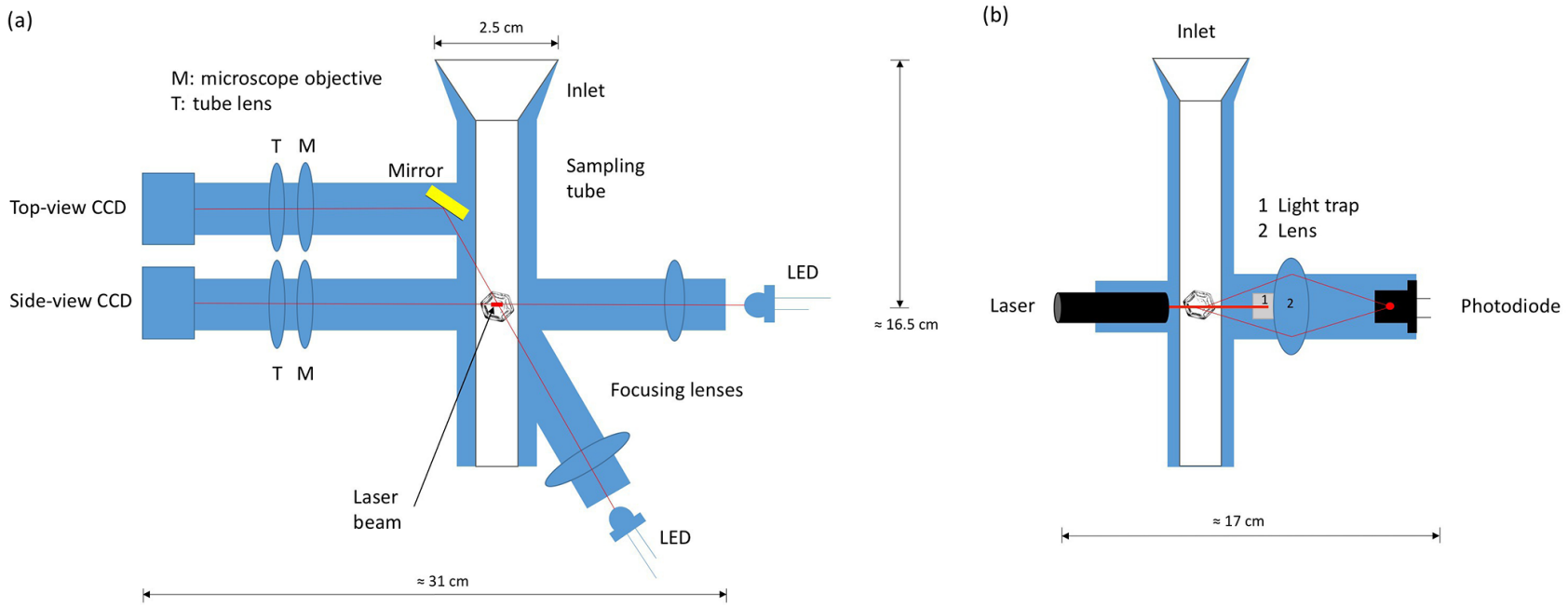

Figure 1. Schematic cut views of the setup of D-ICI. (a) Cut through a plane defined by the optical axes of the imaging optics showing inlet, sampling tube, and the side- and top-view imaging optics and illumination; (b) perpendicular cut showing laser detection consisting in laser, light trap, lens for collection of scattered light, and photodiode. In both panels, the optical cell with the sensing volume at its centre is indicated by the image of an ice crystal (not to scale).

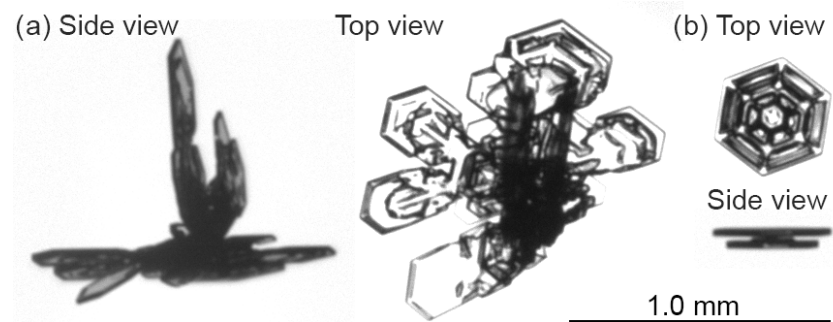

Figure 2. Two examples of ice crystals imaged in two viewing geometries: top view and side view. The ice crystal shown in panel (a) has a width of approximately $1.2 \mathrm{~mm}$; the one in panel (b) has a width of $0.4 \mathrm{~mm}$. Both ice crystals in panels (a) and (b) use the same scaling; for reference, a size bar with length corresponding to $1 \mathrm{~mm}$ (and width of $10 \mu \mathrm{m}$ ) is shown.

detection, the ice particle is optically imaged from two different directions. Figure 2 shows examples of the pairs of images resulting for each ice particle.

One of the two viewing directions looks horizontally from the side, called the side view, and the other vertically from the top, called the top view. The former will allow to measure the fall speed, if using a double exposure (see Sect. 3.3). The latter will provide area and shape as seen in the vertical direction, which are more relevant for fall speed and radiative properties. Because particles fall vertically, an exact vertical viewing geometry for the top view is difficult to achieve, as part of the optics would obstruct the particles' motion. Therefore, the top view is a near-vertically viewing configuration that looks through the optical cell inside the vertical sampling tube at a shallow angle to the vertical axis $\left(17^{\circ}\right)$. The side view, on the other hand, uses exactly a horizontal view-

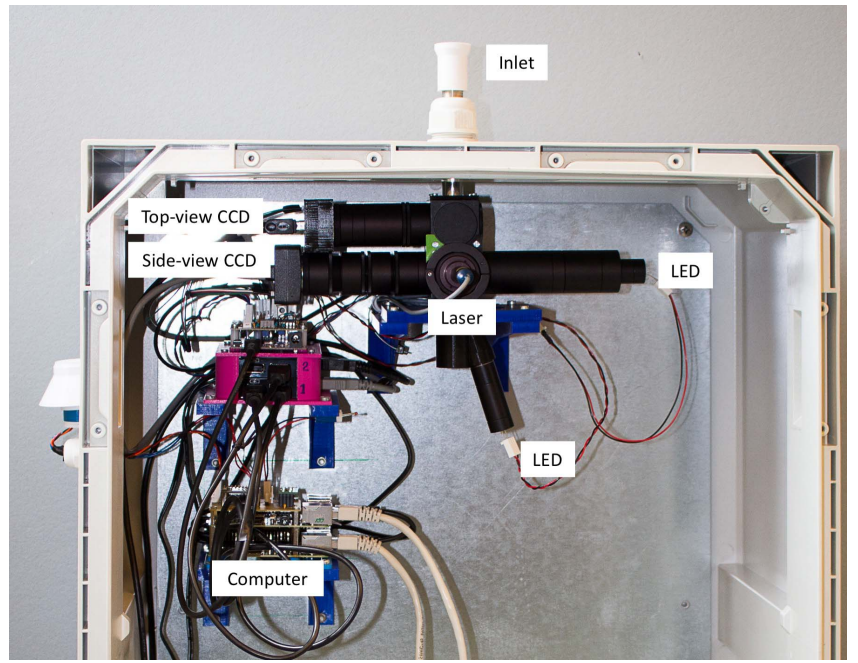

Figure 3. Photograph of D-ICI (door of enclosure is removed).

ing geometry. Figure 3 shows a photograph of D-ICI, and a more detailed description is given in the following sections.

\subsection{Inlet and sampling tube}

Similarly to the ICI probe (Kuhn and Gultepe, 2016), also DICI has a funnel-shaped inlet, wider at the top, with a sharp upper edge (see Figs. 1 and 3). Ice particles fall freely into this inlet. The inlet has a diameter of $25 \mathrm{~mm}$ at the top and narrows down to an inner diameter of $8 \mathrm{~mm}$. It is concentrically mounted atop of the vertical sampling tube with inner diameter of $12 \mathrm{~mm}$. Ice particles falling through the inlet are therefore transferred into the sampling tube. After falling 
about $160 \mathrm{~mm}$ vertically through the sampling tube, ice particles come to the section containing the sensing volume. The length of the sampling tube upstream of the sensing volume is sufficient (more than 10 times the diameter of the sampling tube) so that particles can relax from motion induced by the wind and surface turbulence. However, higher wind speeds may disturb fall speed measurements inside the sampling tube. Hence, the fall speed of ice particles is not affected by light wind or turbulence as the sampling tube is shielding against them, whereas at higher wind speeds one should use caution. Also, the collection efficiency of the inlet will be affected by higher wind speeds, as observed for snow gauges (Goodison et al., 1998), so that snowfall rate and concentration measurements, which will be discussed in Sect. 3.1, become more uncertain with stronger wind speeds.

\subsection{Imaging optics}

In the sensing volume (see Sect. 2.4), particles are optically imaged by two imaging systems, each using a monochromatic CCD camera (Chameleon 1.3 MP Mono USB 2.0, Point Grey, now FLIR) having a $1280 \times 960$ pixel sensor chip with pixels that are $3.75 \mu \mathrm{m} \times 3.75 \mu \mathrm{m}$ in size. These camera systems are similar to the microscope optics used in ice crystal imaging setups with single imaging systems (Kuhn et al., 2012; Kuhn and Gultepe, 2016). They consist of a microscope objective followed by a tube lens, as indicated in Fig. 1. For the horizontal view, i.e. side view, the microscope objective (RMS4X, Thorlabs) has a focal length of $45 \mathrm{~mm}$. For the top-view system, the objective is a single convex lens, a positive achromatic doublet (AC254-050-A, Thorlabs) with focal length of $50 \mathrm{~mm}$. This has, compared to the microscope objective, a longer working distance of $43 \mathrm{~mm}$, which is required for the top-view configuration.

The tube lens of the side-view optics is a positive achromatic doublet (AC254-045-A, Thorlabs) with the same focal length as its microscope objective $(45 \mathrm{~mm})$. As tube lens of the top-view optics, the same achromatic doublet as for its objective is used. Thus, the resulting magnifications are the same for both systems $(M=1)$. Both camera systems have therefore a pixel resolution, i.e. the size of a feature of the imaged object that appears on the image as one pixel, equal to the pixel size of $3.75 \mu \mathrm{m}$. Additionally, the field of view (FOV) is equal to the exposed sensor area, i.e. $4.8 \mathrm{~mm} \times 3.6 \mathrm{~mm}$.

Both imaging systems use bright-field illumination from the back. This is achieved by a light-emitting diode (LED) with a simple focusing lens optics allowing for an even illumination of the FOV. Each of these two lens-LED configurations is arranged along the optical axis of the respective imaging optics on the opposite side of the sensing volume (see Fig. 1). While this illumination scheme reveals some details of the inner structure for most snow particles, due to orientation or particle complexity, some parts of the particle can become opaque for the illumination (see, for example, the particles in Fig. 2). This can be considered a limitation of the current illumination setup. However, the details that can be seen on one or both of the high-resolution images (top and side view) will allow shape classification in most cases (Vázquez-Martín et al., 2020).

The top-view optical system uses a mirror between the sensing volume and the objective lens. This allows to fold its optical axis so that it is parallel to the optical axis of the side-view system for a simpler mechanical setup.

\subsection{Detection and sensing volume}

The sensing volume, i.e. the volume in which particles are detected and imaged, is defined as the intersection of the laser beam for detection with the overlapping FOVs of the imaging systems. The laser beam, which has a wavelength of $650 \mathrm{~nm}$ and power of $1 \mathrm{~mW}$, is aligned perpendicular to the optical axes of both imaging optics. It is shaped by an aperture to about $1 \mathrm{~mm}$ horizontal width, which corresponds approximately to the depth of focus of the side-view camera. The laser beam is further shaped by a cylindrical lens (LJ1960L1, Thorlabs) with focal length of $20 \mathrm{~mm}$ such that its vertical height, originally about $3 \mathrm{~mm}$, is focused to approximately $0.1 \mathrm{~mm}$ in the centre of the FOV of the side-view camera. Thus, the laser beam forms a light sheet with width of approximately $1 \mathrm{~mm}$ and height of $0.1 \mathrm{~mm}$. Both the sideand top-view cameras are focused so that their focal planes are aligned with this resulting laser sheet. As a consequence, all detected particles are in focus for both images.

To determine the snowfall rate or the snow crystal number concentration, the sensing area, i.e. the area through which detected particles fall, needs to be known rather than the sensing volume. The sensing area is the horizontal cross section of the sensing volume (i.e. the cross section perpendicular to the vertical falling motion). The area is therefore given by the product of the width of the FOV of the cameras and the sum of laser beam width $(1 \mathrm{~mm})$ and particle size. This sum has to be used instead of laser beam width only, because particles that are only partially in the laser beam will be detected too. Thus, the sensing area is size dependent (larger particles have a larger sensing area). When assuming a constant sensing area corresponding to a particle size of $500 \mu \mathrm{m}$, the concentrations of particles larger than this size would be overestimated. This overestimation is compensated by the size-dependent probability of a particle to touch one of the image borders. Larger particles are more likely to touch an image border, i.e. to be partially outside the image. Such ice particles that touch one image border are therefore excluded from data analysis (see Sect. 3.2). This exclusion from further analysis results in an underestimation of larger particles, hence compensating the overestimation due to sizedependent sensing area. Thus, the assumption of a constant sensing area does not cause a significant uncertainty, as was also discussed by Kuhn and Gultepe (2016), and the sensing area to be used is $4 \mathrm{~mm} \times(1 \mathrm{~mm}+500 \mu \mathrm{m})=6 \mathrm{~mm}^{2}$. Here, 
we use $4 \mathrm{~mm}$ as FOV instead of $4.8 \mathrm{~mm}$ mentioned earlier due to the fact that the FOV of the top-view camera is somewhat restricted as a consequence of incomplete illumination of the whole camera FOV (see Sect. 3.2 and Fig. 4 for an example of a complete image).

Scattered light from the part of the laser beam within the sensing volume is collected and focused on a photodiode (FDS010, Thorlabs) by two plano-convex detector lenses (LA1951-A, Thorlabs). The photodiode is located along the axis of the laser beam, which is stopped by a light trap mounted in the centre of the first lens. The diameters of the light trap and the lens tube holding the detector lenses are such that the photodiode detects light scattered by ice particles in the sensing volume in near-forward direction in the range of scattering angles between approximately 10 and $32^{\circ}$. The photodiode has a circular sensitive area with a diameter of $1 \mathrm{~mm}$. Its small area means that most particles that are outside the sensing volume, but still in the laser beam, do not scatter light that can be detected by the photodiode. This minimizes false triggers; i.e. detected scattering leading to empty images as particles are outside the FOVs of the two cameras.

The current of the photodiode is converted to a voltage and amplified (effective current-to-voltage amplification of $2.2 \mathrm{M} \Omega$ ). The resulting photodetector voltage, proportional to the scattered light's intensity, is compared to a threshold voltage (approximately $0.15 \mathrm{~V}$ ). A trigger signal is issued whenever the photodetector voltage is larger than this threshold. The trigger signal is used to trigger the two images to be taken of the detected ice crystal as well as the two background-illuminating LED flashes. Hence, all particles larger than a certain threshold size are detected and then imaged. With the help of Mie scattering calculations (see, e.g. Bohren and Huffman, 1983) this threshold size (diameter of spherical ice) can be estimated as approximately $10 \mu \mathrm{m}$.

\subsection{Computer and data collection}

Both imaging systems are triggered by the same signal (see Sect. 2.4). To guarantee simultaneous imaging by the two cameras, each of the two imaging systems has its own dedicated computer for operation and data collection. In this way, there are no particular requirements about the computer's performance, and two Raspberry Pi devices are used for DICI. Each computer stores its own image data on an SD card, which is connected to the computer's USB port via a card reader. One of the two computers acquires also temperature inside and outside the instrument, registered by two thermistors, and the outside relative humidity with a HIH-4000 sensor (Honeywell) with an accuracy of $\pm 3.5 \%$.

Both computers are connected to a network via ethernet cables. This allows to synchronize them with each other. Consequently, corresponding side- and top-view images can be recognized by their time stamp, which is part of the file name. Both computers can be accessed through an additional laboratory or office computer, which is connected to the same network via cable or internet, if the network provides internet access. Data can then be retrieved using this laboratory computer.

Alternatively, the SD cards can be collected to copy the image data. Then, the image data will be processed by the laboratory computer as described in Sect. 3.2.

\section{Methods}

\subsection{Snowfall rate and number concentration}

While the focus of D-ICI is high-resolution images for shape and fall speed measurements, snowfall rate and number concentration can also be determined from the acquired data.

For that, snowfall rate $r_{\mathrm{s}}$ is defined here as number of snow crystals falling on a given area during a given sampling time $t$. The inlet is sampling falling snow crystals from a larger area than the cross section of the sampling tube, which results in an enhanced number of snow crystals in the sampling tube. To account for this enhancement, an effective sensing area $A$ is used. It is larger than the sensing area by a factor equal to the ratio of the areas of the $25 \mathrm{~mm}$ inlet and the $12 \mathrm{~mm}$ sampling tube, i.e. a factor of 4.3. This yields $A=4.3 .6 \mathrm{~mm}^{2}=26 \mathrm{~mm}^{2}$. Then, $r_{\mathrm{s}}$ is determined as number of snow crystals $N$ divided by the effective sensing area $A$ and sampling time $t$ :

$r_{\mathrm{s}}=\frac{N}{A t}$.

The number concentration $n$ is calculated from $N$ divided by the sampling volume $V$. To determine $V$, a constant fall speed $v$ of $0.5 \mathrm{~m} \mathrm{~s}^{-1}$ is assumed, which corresponds approximately to the average fall speed of the data used here. With this assumption, the effective sampling flow rate of DICI becomes $A v=13 \mathrm{~cm}^{3} \mathrm{~s}^{-1}$. Finally, $n$ is calculated using Eq. (2).

$n=\frac{N}{V}=\frac{N}{A v t}$

The size dependencies of the sensing area and the probability of the particle being partially outside the FOV cancel out to a good approximation (see Sect. 2.4). This size dependency may be corrected, and the correction factor for number concentration would vary between 1.07 and 1.09 for particles with maximum dimensions between 1.0 and $2.0 \mathrm{~mm}$, reaching down to a minimum of 1.03 for particles of $1.4 \mathrm{~mm}$. For particles down to 0.5 or up to $2.5 \mathrm{~mm}$, it would increase to approximately 1.25 .

The assumption of constant fall speed $v$ of snow particles, mentioned above, introduces a new uncertainty. When the constant speed of $0.5 \mathrm{~m} \mathrm{~s}^{-1}$ is overestimating the actual particle fall speed, the concentration $n$ of these particles is underestimated. And conversely, underestimating the 
speed results in overestimating concentration. About twothirds of the data used here had fall speeds between 0.3 and $0.85 \mathrm{~m} \mathrm{~s}^{-1}$; this means that for those particles the error in concentration ranges from, respectively, underestimating concentration by about $40 \%$ to overestimating it by $70 \%$. This may be corrected for with correction factors based on measured fall speed, which would then vary for the twothirds of data considered here between about 1.7 and 0.6 , respectively.

An additional uncertainty in estimating the effective sensing area resulting from the uncertainty in determining the laser beam width, which may be on the order of $\pm 20 \%$, however, is difficult to measure.

These uncertainties affect both $n$ and $r_{\mathrm{s}}$. We have not yet verified the uncertainties experimentally. Also, wind speed, likely affecting these measurements (Goodison et al., 1998), has not been considered yet. Hence, $n$ and $r_{\mathrm{s}}$ determined with D-ICI using the assumptions and estimates outlined above should be considered estimates of the actual number concentration and snowfall rate.

\subsection{Image processing}

The images have pixels with grey levels between 0 (black) and 255 (white). An automated image processing algorithm is applied to all top-view images to retrieve ice particle size, area, area ratio, and aspect ratio. It first removes non-particle features from the background. Then, the particles on the images are detected and their edges are found. This algorithm has been used by Kuhn and Gultepe (2016); Vázquez-Martín et al. (2020) and is a simplified implementation in Matlab of the algorithm described in Kuhn et al. (2012). Here, we summarize this implementation briefly. In the following, the different steps of the algorithm are described, of which some are shown in Fig. 4 for an example image.

A background image without any ice particle is used to correct for uneven background illumination, i.e. remove nonparticle features from the background. For this, the difference between background and image to be analysed is determined. The difference is positive where the presence of a particle makes the image darker than the background. For regions where the image is brighter than the background, the resulting negative values are set to zero. These are usually only regions within an ice particle where transmitted light can appear as a brighter spot, surrounded by darker features or the edge of the particle. Now, images are rejected from further analysis if no particle was captured on them, i.e. images that are very similar to the background. For this, a lower threshold is applied to the difference. The image is rejected if the difference does not exceed the threshold for any pixel. A suitable threshold is 30 ; images with ice particles exceed this by a large margin.

Then, for the remaining images, the difference to the background is first scaled to increase the dynamic range of grey values. This is done for each pixel individually, so that

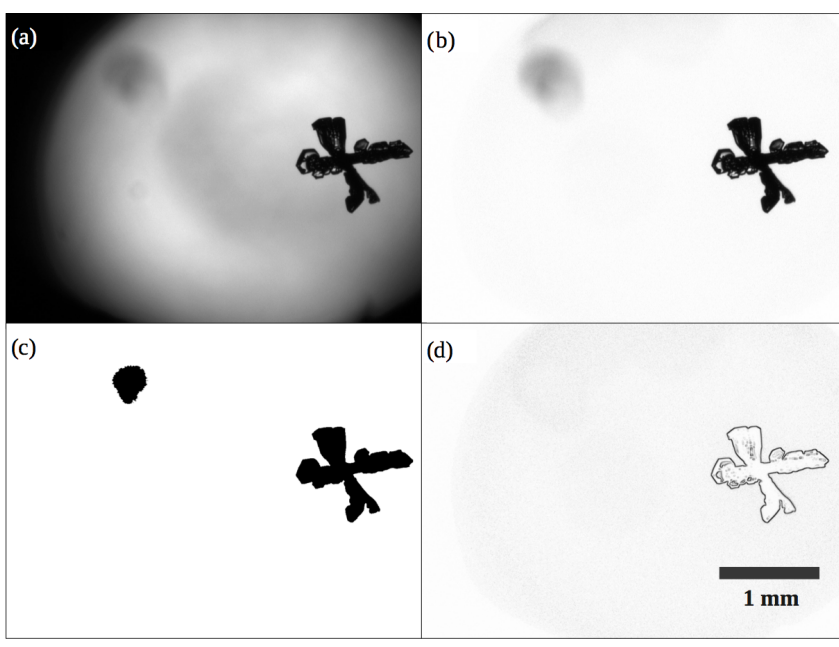

Figure 4. Automated image processing steps shown for an example image. Panel (a) shows the original image; (b) cleaned image (background features removed); (c) binary mask, where logical True values correspond to regions on the cleaned image that are darker than the grey-level threshold, here shown as black; (d) gradient matrix computed from the cleaned image, with values scaled to grey levels for representation (largest gradient value corresponds to black and zero gradient to white). See description in text for details of the processing procedure. The resolution is indicated by a size bar of $1 \mathrm{~mm}$.

the possible maximum difference (when the image pixel is black), at any background pixel becomes 255 . Effectively, the scaling factor at any pixel is $255 / \mathrm{bg}$, where bg is the grey level of the corresponding background pixel. To avoid large scaling factors where the background is dark (bg is small), the factor is limited to 2.5 . For very dark background ( $b g<20)$ the scaling is set to 1 . This scaled difference is then inverted by subtracting it from 255 , so that the resulting greylevel image represents the image cleaned from background features. This can be seen for an example image in Fig. 4, where Fig. 4a shows the original image and Fig. $4 \mathrm{~b}$ the image after the background has been removed. Regions of the original image that were identical to the background or had brighter spots are now white (255) in this cleaned image, and regions where the original image was darker than the background show now grey levels $(<255)$.

The following steps in the image processing apply to the cleaned image resulting from the background removal described above. For detecting in-focus particles, two thresholds are applied: a grey-level threshold and a gradient threshold. The grey-level threshold is used to find particles and their edges, and the gradient threshold is used to reject outof-focus particles. First, images that do not have any pixel darker than the grey-level threshold are discarded. This rejects particles that are much out of focus. Then, a binary mask, i.e. a black-and-white image, of the same dimension as the original image is created where logically True entries represent image pixels that are darker than the grey-level thresh- 
old. The binary mask is then smoothed to remove variations at the 1-pixel level, which are considered to not reflect the actual variations in the edge of the ice particle. The smoothing is achieved by first dilating each True pixel in the binary mask so that the four neighbouring pixels (above, below, right, and left) will also be True. Then, the dilated binary mask is eroded, to restore its original size, by setting the four neighbours of each False pixel to be also False. Between the dilation and erosion steps, the binary mask is also filled, i.e. all pixels that are False but completely enclosed by True pixels are converted to True. This will include the brighter spots, which many ice crystals show on the images, to the particle they belong to. Then, on the resulting black-and-white image (see example in Fig. 4c), ice particles are represented by connected True pixels in the binary mask. All particles, i.e. regions of connected pixels that are now included in this binary mask, are then identified and their edges are found (with the Matlab function bwboundaries). For each particle, this results in both a list of coordinates of the edge pixels and a mask containing all pixels that belong to the particle. Each particle can then be processed individually.

Firstly, out-of-focus particles are rejected. For this purpose, a gradient matrix is computed from the image. The values of this matrix are used as a parameter indicating in-focus or out-of-focus particles. For computing the gradient values, the image is filtered (using the Matlab function imfilter) with a Sobel horizontal edge-emphasizing filter (generated with the Matlab command fspecial( "sobel")) and its transpose, i.e. with the corresponding vertical filter. The resulting matrices represent the horizontal and vertical gradients, respectively. The values of the gradient parameter are then calculated as the sum of the absolute values of these horizontal and vertical gradients (Kuhn et al., 2012). For each particle, the maximum gradient value of all pixels associated with that particle is then compared to the gradient threshold. The particle is rejected as out of focus if this maximum is lower than the threshold. For the example image of Fig. 4, two ice particles are found using the grey-level threshold (see Fig. 4c); however, one of these two particles is rejected based on the low values in the gradient matrix (shown in Fig. 4d).

Secondly, particles with apparent problems are marked with quality flags. A particle that is in part out of focus can sometimes have parts of the edge not being detected, yielding an apparently fragmented edge with narrow gaps. Similarly, if thin ice particle features result in brighter pixels than the grey-level threshold, a fragmented edge is the consequence. To account for this, two or more detected particles that appear very close to each other are joined and the resulting particle is marked as being "fragmented". The area of such a particle as determined from the detected pixels will be too small. The resulting error is not large, because the gaps are only small, and by joining the fragmented pieces, the particle may still be considered. However, being marked, it can also easily be excluded from further analysis. An example of an ice particle detected with fragmented edge is given in Fig. 5b).
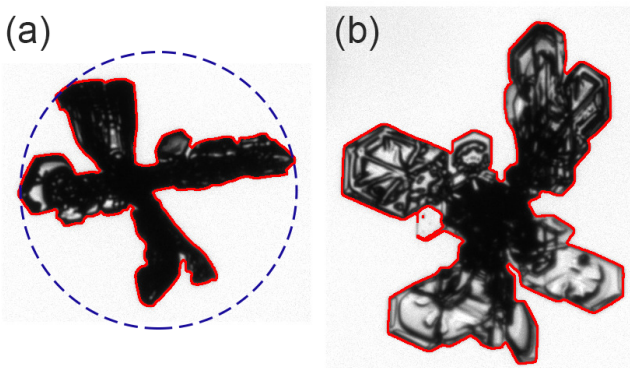

Figure 5. Detected edges of processed ice particle images. The edges are shown in red and have been enlarged to a thickness of 3 pixels for better visibility in this figure. One example, panel (a) shows the edge of the ice particle from Fig. 4. The smallest circle enclosing the particle is shown with a dashed line; its diameter, i.e. the maximum dimension of the ice particle, is $1.34 \mathrm{~mm}$ (or 358 pixels). The other example in panel (b) shows an ice particle that has been detected with fragmented edge due to parts of the actual particle edge being too bright (see text for more details).

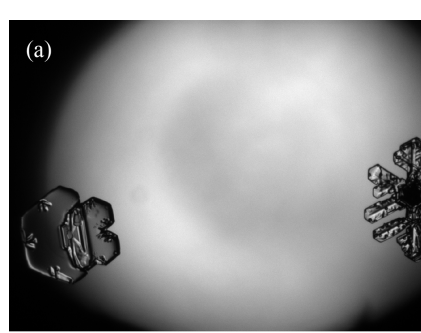

(b)
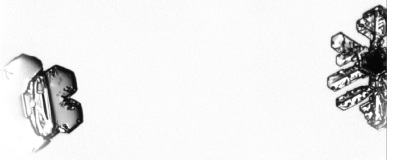

Figure 6. Examples of ice particles flagged as"on-border" (on the right side of the image) and "in-darkregion" (on the left side of the image). The original image (a) and the image after background removal (b) are shown.

The other ice particle in the same figure shows the unfragmented edge of the example particle from Fig. 4. In addition, particles that are touching the image border are marked with another flag as "on-border". Their size and area are underestimated as they are in part outside the image. Thus, using this flag, they can be excluded from analysis when size and area matter. Figure 6 shows an example of an ice particle with the "on-border" flag. A further problem is related to incomplete illumination of the top-view images due to restricted geometry in the longer light path in top-view compared to side-view optics. This results in dark corners where ice particles cannot be seen. Consequently, also particles touching these dark corners have to be excluded from analysis as their size cannot be known, similarly as for "on-border" particles. To allow this, these particles are marked with an additional flag as "in-darkregion" when they have at least 1 pixel within the dark corners. For this, a mask containing the corresponding dark pixels (darker than a certain threshold) in the corner regions is constructed from the background image. Figure 6 shows an example of an ice particle with the "in-darkregion" flag. 
Lastly, area and size information is determined for each detected ice particle. As a parameter describing a characteristic size of the detected particle we are using maximum dimension, i.e. the diameter of the smallest circle that completely encloses that particle on the image (see Fig. 5 for an example). The area corresponds to the number of pixels that represent the particle in the binary mask. Both size and area are given in units of pixels. They are then converted to actual length and area by multiplication with the pixel resolution and squared pixel resolution, respectively.

As this method is the same as used for the imager described by Kuhn et al. (2012), which used similar optics, sizing accuracy is expected to be similar. There, the determined size of a small particle (about 50 pixels in size) varied by about 2 pixels when the location within the depth of focus was changed. Larger inaccuracy is avoided by rejecting out-of-focus particles. To this uncertainty, 1 pixel should be added to account for uncertainty of particle edge location. Thus, a combined sizing accuracy of approximately $10 \mu \mathrm{m}$ (or 3 pixels) is expected for D-ICI. Consequently, for a $200 \mu \mathrm{m}$ particle, the expected error in area should be on the order of $10 \%$.

\subsection{Fall speed measurement}

The side-view camera can be operated in a fall speed mode, in which the falling ice particle is captured twice on the same image by using a double exposure. This concept has been tested with ICI in a configuration without inlet, so that ice particles could freely fall through the instrument (Kuhn and Gultepe, 2016). For D-ICI, the inlet and sampling tube are designed so that particles fall vertically undisturbed before they reach the sensing volume; thus, the setup does not need to be modified to allow measurements of fall speed. In the fall speed mode, two very short illumination flashes are used, which have a time separation of $\Delta t=1.26 \mathrm{~ms} \pm 0.01 \mathrm{~ms}$. This time difference is long enough to yield a clear separation of the two particle appearances on the image, but also short enough so that the particle does not fall out of the vertical FOV of the imaging optics. Thus, the particle's fall speed $v$ can be determined from the vertical fall distance $\Delta s$, as measured on the image, and the time separation $\Delta t$ of the two exposure flashes simply as

$v=\frac{\Delta s}{\Delta t}$.

The vertical fall distance $\Delta s$ is measured by manual inspection of the side-view images. Two or three points at extremes of each particle to be analysed (e.g. a far right corner and far left corner point) are identified and their coordinates on the image are recorded. The same points are then also identified and recorded on the second appearance of the particle on the image, and the vertical distance is determined as the difference of the vertical coordinates of pairs of corresponding points of the two appearances. From the two or three vertical distances determined in this way, an average vertical fall distance is calculated.

While falling, the difference of the horizontal coordinates is usually close to zero. Such a difference could be caused by sideway or rotating (tumbling) motion. Horizontal winds, which affect other instruments, with an open sampling volume, such as PIP and MASC, do not cause a sideway motion in the enclosed sensing volume of D-ICI. Thus, only a tumbling particle can be responsible for a difference of the horizontal coordinates, and tumbling of ice particles is not often seen (see Sect. 4.2). If it occurs, it is detected by significantly different values of the individual vertical distances measured for a point on the right and left sides of the particle, respectively, so that particles that are tumbling too much may be excluded from analysis of fall speed data. When tumbling, one side of the snow particle falls faster and one slower than the average that is determined from the averaged fall distances $\Delta s$. Thus, by rejecting tumbling particles, e.g. those that rotate by more than $10^{\circ}$, the error in fall speed can be limited to approximately $7 \%$. Uncertainties in $\Delta t$ have a negligible effect on fall speed error. Also, the error related to accuracy of point selection (about 2 pixels), which translates to an additional uncertainty in $\Delta s$, is only on the order of $1 \%$.

While side-view images are not processed automatically, the top-view images are (see Sect. 3.2). Results from this automatic processing of top-view images provide size, area, area ratio, and aspect ratio for the particles, whose fall speeds are determined from the corresponding side-view images.

\section{Measurements}

\subsection{Images and shapes}

According to the design, the pixel resolution should be equal to the pixel size of the CCD cameras, $3.75 \mu \mathrm{m}$ (see Sect. 2.3). This has been confirmed by imaging a calibration target, a graticule with $10 \mu \mathrm{m}$ per division and total length of $1 \mathrm{~mm}$. The lengths in pixels corresponding to $1 \mathrm{~mm}$ from several such images have been converted to pixel resolutions yielding an average of $3.74 \mu \mathrm{m}$ pixel $^{-1}$ with a standard deviation of $0.02 \mu \mathrm{mixel}^{-1}$ for the side-view imaging optics, and, from separate images, the same values for the top view.

Figure 7 shows a few examples of ice particle images from snowfall in early winter (23 October 2014 in Kiruna), when the ambient surface temperature was about $-5^{\circ} \mathrm{C}$. Each ice particle is shown in the two views, where the top view is shown above the corresponding side view.

These detailed images of ice particles allow to recognize their shapes. On 23 October 2014, the ice particles had predominantly bullet-rosette and similar shapes, but also platelike and capped-column shapes (see Fig. 7). On another day, 19 October 2014, with similar ambient surface temperatures of about -3 to $-6^{\circ} \mathrm{C}$, two dominant shapes were observed: graupel (heavily rimed snow crystals) and rimed needles (see 

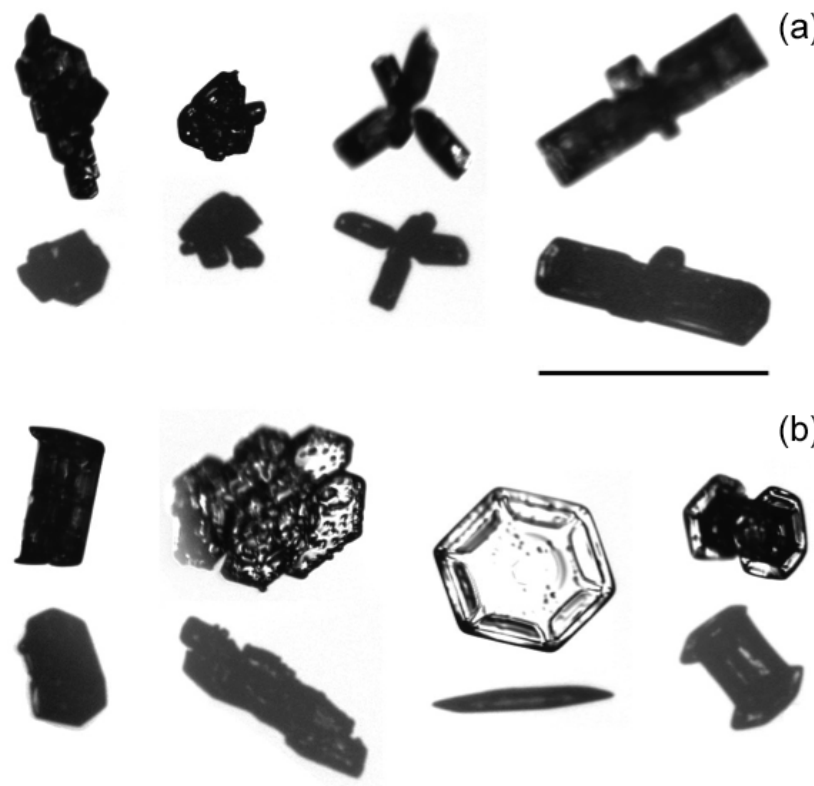

Figure 7. Ice particles as imaged in two viewing geometries: top view and side view. Each ice particle is shown as a pair of these two views, with the top view always above the corresponding side view. Eight particles are shown in two rows $(\mathbf{a}, \mathbf{b})$ of such pairs. All images have the same resolution; for reference, a size bar with length corresponding to $1 \mathrm{~mm}$ is shown.

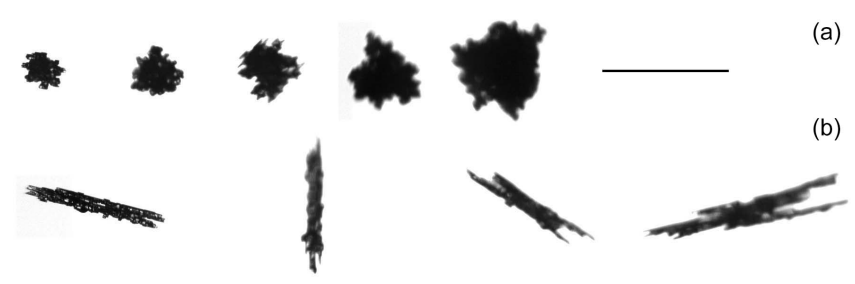

Figure 8. Example images of the two shapes, graupel (a) and rimed needles (b), from the snowfall measured on 19 October 2014. For reference, a size bar with length corresponding to $1 \mathrm{~mm}$ is shown.

Fig. 8). Most of the rimed needles on that day seemed to be agglomerates or ensembles of two or more single needles (called bundles of needles by Magono and Lee, 1966).

\subsection{Fall speed}

Figure 9 shows examples of double-exposed images from the side view, showing the falling ice particles twice, used to determine fall speed. The data considered in the following are from 19 October 2014, a day with relatively low wind speeds with on average $2 \mathrm{~m} \mathrm{~s}^{-1}$ (as measured at the nearby Kiruna airport). Therefore, we do not consider these data to be affected much by issues related with higher wind speeds. The images from 19 October 2014 (Fig. 9a) also include a few drizzle droplets. The heavy riming on that day indicates the presence of cloud droplets, and the imaged drizzle droplets

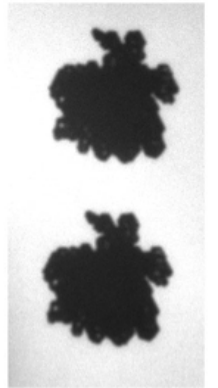

(a)
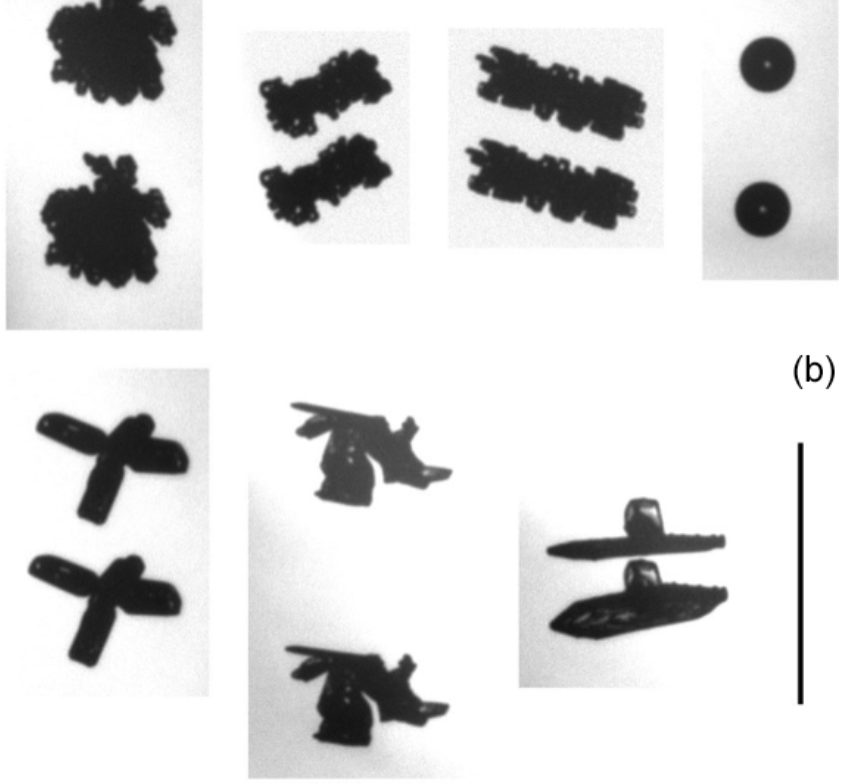

(b)

Figure 9. Example side-view images of doubly exposed falling ice particles. The fall speed is determined from the vertical separation of the two instances of the particle on the same image. Panel (a) shows measurements from 19 October 2014; panel (b) shows measurements from 23 October 2014. For reference, a size bar with length corresponding to $1 \mathrm{~mm}$ is shown.

originate from such cloud or fog droplets that have grown large enough to precipitate and fall into the inlet of D-ICI. They were, with only very few exceptions, smaller than all snow particles.

One of the particles shown in Fig. 9b is tumbling (rightmost ice particle). The rotation, around an axis perpendicular to the image plane, of the particle between the two exposures is approximately $8^{\circ}$, which still seems acceptable if one wants to determine fall speed with an error of below about $10 \%$. Hence, $10^{\circ}$, or perhaps up to $15^{\circ}$, may be used as limit, above which the image has to be discarded for fall speed measurement. Selecting a few days randomly and analysing the ice particle images on those days (total of 946 particle images) yields that approximately $8 \%$ of ice particles are tumbling by more than an angle of $10^{\circ}$, and only $3 \%$ more than $15^{\circ}$. This means that particles in general tumble somewhat; however, the majority of ice particles tumble so little in the time between the two side-view exposures that fall speed can still be measured.

\subsection{Cross-sectional area}

Using the top-view images, the ice particles' projected area in the fall direction (i.e. area projected on a surface perpendicular to the vertical fall direction) can be determined. Figure 10 shows these projected, or cross-sectional, areas $A$ 


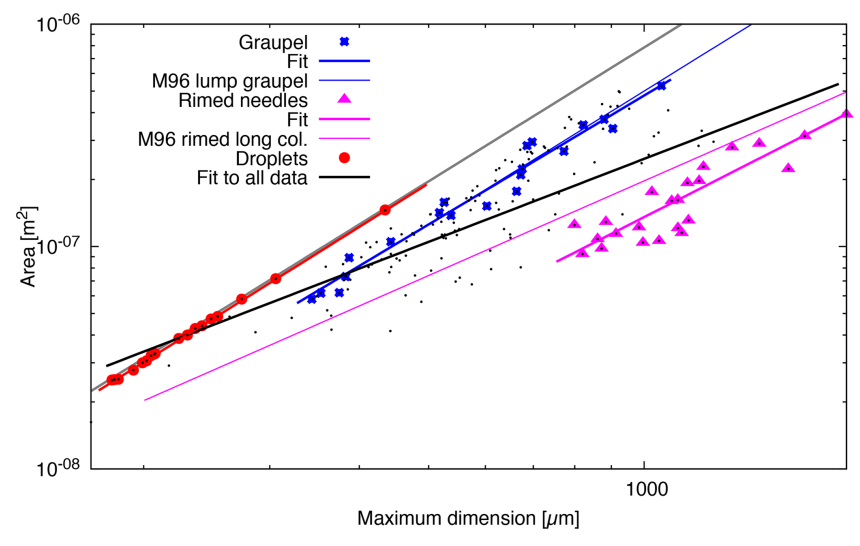

Figure 10. Area $A$ versus maximum dimension $D$ from snowfall measured on 19 October 2014 between approximately 06:00 and 19:00 UTC. Fits to all data (black dots) and to three subsets corresponding to graupel, rimed needles, and droplets are shown as lines in the same colour as the corresponding data points. For comparison, a grey line indicates the cross-sectional area of spheres. In addition, two relationships reported by Mitchell (1996, referred to as M96 in the legend) are shown as thinner lines: one for rimed long columns (magenta) and one for lump graupel (blue).

from snowfall measured on 19 October 2014 between approximately 06:00 and 19:00 UTC (at temperatures on the ground between -3 and $-6^{\circ} \mathrm{C}$ ) as a function of particle size, i.e. maximum dimension $D$, also determined from the topview images. On this logarithmic plot, the cross-sectional area of spheres having a diameter equal to the maximum dimension is represented by a straight line given by $A=$ $\pi / 4 \cdot D^{2}$. A power law $A=\gamma D^{\beta}$ can be fitted to the data to find the parameters $\gamma$ and $\beta$.

For the data shown in Fig. 10, this yields $A=4.72$. $10^{-11} \mathrm{~m}^{2} \cdot D^{1.24}, D$ in $\mu \mathrm{m}$, with a correlation coefficient $R^{2}=$ 0.71 .

When the ice particles are classified according to their shapes, power laws can be fitted to the resulting subsets of data to find relationships describing area for specific shapes. On 19 October 2014, two dominant shapes were observed: graupel and rimed needles (see Fig. 8). The fitted power laws for these two shapes are indicated in Fig. 10 by coloured lines and are given by

graupel : $\quad A=7.89 \cdot 10^{-13} \mathrm{~m}^{2} \cdot D^{1.93}, \quad\left(R^{2}=0.97\right)$

rimed needles : $\quad A=1.63 \cdot 10^{-12} \mathrm{~m}^{2} \cdot D^{1.64}$,

$$
\left(R^{2}=0.78\right) \text {, }
$$

with $D$ in $\mu \mathrm{m}$. The groups of particles used for these fits are shown in Fig. 10 as coloured symbols and correspond to a selection of the most compact-looking graupel and almost all particles that could be identified as rimed needles.

The images from 19 October 2014 also show a few drizzle droplets, which can be seen in Fig. 10 with areas very close to the area-dimension relationship for spheres. Droplets are the

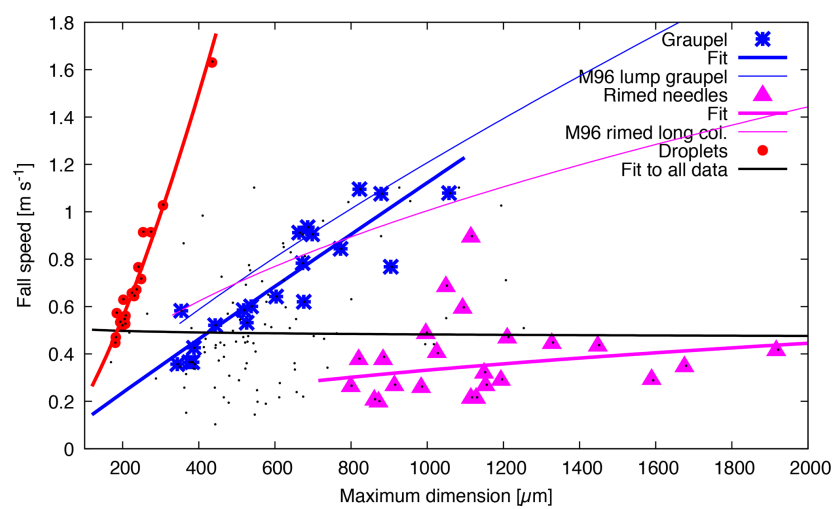

Figure 11. Fall speed versus maximum dimension $D$ for snowfall measured on 19 October 2014 between approximately 06:00 and 19:00 UTC. Fits to all data (black dots) and to three subsets corresponding to graupel, rimed needles, and droplets are shown as lines in the same colour as the corresponding data points. In addition, two relationships predicted from area and mass relationships using the method reported by Mitchell (1996, Eqs. 12 and 20, referred to as M96 in the legend) are shown as thinner lines: one for rimed long columns (magenta) and one for lump graupel (blue).

smallest particles measured by D-ICI on that day, with maximum dimensions of below $200 \mu \mathrm{m}$ for the smallest droplets. Due to their spherical shapes, the determined area ratios were very close to 1 , and all particles with area ratio larger than 0.9 were droplets. For these, the fitted area-dimensional power law is $A=6.79 \cdot 10^{-13} \mathrm{~m}^{2} \cdot D^{2.02}$ ( $D$ in $\mu \mathrm{m}, R^{2}=1.00$ ), which is very close to the cross-sectional area of spheres.

When looking at the area-dimensional relationship for a certain shape, the fit to the power law can be very good. An exception here are rimed needles. However, they still have a fairly good fit, better than the fit to all data with one common power law, which would predict poorly the area for any of the shapes here, droplets, graupel, and rimed needles (see Fig. 10). Figure 10 also shows for comparison two relationships reported by Mitchell (1996): one for rimed long columns (as a thin line in magenta) and one for lump graupel (blue). While the latter agrees very well with our graupel, the rimed long columns have a larger cross-sectional area than our rimed needles, which one would expect for columns compared to thinner needles.

\subsection{Fall speed measurements}

Figure 11 shows the fall speeds versus the maximum dimension of individual ice particles from the snowfall measured on 19 October 2014. The spread of the data is considerable, and fitting to a power law of the form $v=c D^{b}$ yields $v=0.55 \mathrm{~m} \mathrm{~s}^{-1} \cdot D^{-0.019}$ ( $D$ in $\mu \mathrm{m}$ ) with $R^{2}=0.0004$; i.e. no dependence of speed on size is found, indicated by the exponent $b$ and $R^{2}$ being close to zero. The parameter $c$ coincides with the average fall speed of $0.55 \mathrm{~m} \mathrm{~s}^{-1}$. As mentioned in Sect. 4.3, the dominant shapes on that day were graupel 
and rimed needles. Using the subsets of the data representing these two shapes, now fits to the power law reveal significant correlations for graupel. However, for rimed needles, the power law does not fit the data well. The results from these fits are

$$
\begin{gathered}
\text { graupel : } \quad v=0.0013 \mathrm{~m} \mathrm{~s}^{-1} \cdot D^{0.98}, \quad\left(R^{2}=0.83\right), \\
\text { rimed needles : } \quad v=0.020 \mathrm{~m} \mathrm{~s}^{-1} \cdot D^{0.41}, \\
\left(R^{2}=0.054\right),
\end{gathered}
$$

with $D$ in $\mu \mathrm{m}$. These fitted power laws are shown in Fig. 11 as solid lines. The relationships for area (see Fig. 10) and mass reported by Mitchell (1996) can be used to derive the corresponding fall speed (Mitchell, 1996, Eqs. 12 and 20). The resulting relationships for rimed long columns and one for lump graupel are shown in Fig. 11 for comparison. As for area, the relationship for lump graupel agrees well with the D-ICI graupel measurements, whereas there are differences for rimed long columns compared to our rimed needles. These discrepancies are probably related to the larger area and mass of columns compared to needles.

The figure also shows the fall speed measured for the drizzle droplets. As expected, the droplets have the strongest dependence on size. With increasing complexity of particle shape, from droplets over graupel to rimed needles, the size dependence becomes weaker, the spread in data larger, the speed (at same size) slower, and $R^{2}$ of the fit to a power law smaller. Droplets have the simplest shape (spherical), and also the largest area ratio of larger than 0.9. The compact graupel particles that have been selected to fit the fall speed-size relationship have a somewhat lower area ratio, on average 0.63 (with standard deviation of 0.08). Rimed needles have the lowest area ratio of on average 0.17 (SD 0.04). Thus, one can also observe that with decreasing area ratio the size dependence of fall speed becomes weaker and at the same time the fit to the power law worsens. So, if instead of compact graupel all particles with area ratios between 0.25 and 0.9 are selected, a group that includes graupel with more structure and smaller area ratio compared to compact graupel, then we expect the fit quality to deteriorate. And in fact, for this group with an average area ratio of 0.56 (SD 0.15), the results of a fit are $v=0.0079 \mathrm{~m} \mathrm{~s}^{-1} \cdot D^{0.66}, D$ in $\mu \mathrm{m}$ (with $R^{2}=0.20$ ).

\section{Summary}

We have described the Dual Ice Crystal Imager (D-ICI), a ground-based in situ instrument to determine snow ice crystal properties and fall speed simultaneously. Dual images are taken of detected snow particles using two CCD cameras that image along a horizontal and close-to-vertical viewing direction, respectively. The horizontal, or side, view is used to determine fall speed from images taken with double exposures.
The close-to-vertical, or top, view is used to determine size and area.

Both cameras use the same pixel resolution of approximately $4 \mu \mathrm{m} \mathrm{pixel}^{-1}$. The high-resolution images provide enough detail to determine shape in most cases. Having two views of the same particle helps to avoid ambiguities in shape determination that may arise, if only one image were used, due to either an unfavourable particle orientation or particle complexity obscuring internal structure in the current illumination setup. Hence, D-ICI can be used for classification studies (Vázquez-Martín et al., 2020). Microphysical properties may then be studied specifically for certain shapes. The necessity to discriminate shapes has been demonstrated by fitting one common power law for area versus size to all data during a certain measurement period. The relationship that has been found would fit the area poorly for any of the shapes encountered in that period, droplets, graupel, and rimed needles. By selecting subsets of the data corresponding to certain shapes, better fitting relationships have been found and reported (see Sect. 4.3). Similarly, fall speed-size relationships have been found to differ from shape to shape with varying correlations, which, however, are all better than correlation if shape is not considered (see Sect. 4.4). Thus, an instrument that allows to discern shape and measures fall speed at the same time is required.

Snow particles fall some distance vertically through the sampling tube before images are taken, from which speed is derived. Therefore, the fall speed measurements of D-ICI are not affected by the vertical component of the wind speed or by turbulence close to the ground. The accuracy of fall speed measurements has been discussed and is mainly limited by tumbling of snow particles. However, tumbling is not observed frequently. Rejecting particles that tumble with a rotation of more than $10^{\circ}$ as detected on the side-view image, the error can be limited to $7 \%$.

Snow particle size and area are determined from top-view images, i.e. as projected along the vertical fall direction. These properties are more appropriate than the same properties determined from a horizontal view, as done by most instruments, when studying relationships to the fall speed or comparing to vertically pointing remote sensing measurements.

Data availability. The presented data are available at the Swedish National Data Service (SND-ID: SND 1129; https://doi.org/10.5878/rhwc-7093; Kuhn, 2020).

Author contributions. TK designed and built D-ICI. TK prepared the analysis methods with contributions from SV-M. TK carried out the measurements in 2014-2016; SV-M carried out the measurements from 2017 on with contributions from TK. TK analysed data from 2014. SV-M analysed data used to evaluate frequency and ex- 
tent of tumbling. TK prepared the manuscript with contributions in text and figures from SV-M.

Competing interests. The authors declare that they have no conflict of interest.

Acknowledgements. We thank the Kempe Foundations (Kempestiftelserna, SMK-1024) for financial support of hardware, the Swedish National Space Agency (SNSA) for funding during the prototype development (grant Dnr 85/10), and the Graduate School of Space Technology at Luleå University of Technology for additional financial support.

Financial support. This research has been supported by the Kempe Foundations (grant no. SMK-1024) and the Swedish National Space Agency (grant no. Dnr 85/10).

Review statement. This paper was edited by Alexander Kokhanovsky and reviewed by Kevin Hammonds and Timothy Garrett.

\section{References}

Abdelmonem, A., Schnaiter, M., Amsler, P., Hesse, E., Meyer, J., and Leisner, T.: First correlated measurements of the shape and light scattering properties of cloud particles using the new Particle Habit Imaging and Polar Scattering (PHIPS) probe, Atmos. Meas. Tech., 4, 2125-2142, https://doi.org/10.5194/amt-4-21252011, 2011.

Amsler, P., Stetzer, O., Schnaiter, M., Hesse, E., Benz, S., Moehler, O., and Lohmann, U.: Ice crystal habits from cloud chamber studies obtained by in-line holographic microscopy related to depolarization measurements, Appl. Optics, 48, 5811-5822, 2009.

Barthazy, E., Göke, S., Schefold, R., and Högl, D.: An optical array instrument for shape and fall velocity measurements of hydrometeors, J. Atmos. Ocean. Tech., 21, 1400-1416, 2004.

Battaglia, A., Rustemeier, E., Tokay, A., Blahak, U., and Simmer, C.: PARSIVEL Snow Observations: A Critical Assessment, J. Atmos. Ocean. Tech., 27, 333-344, https://doi.org/10.1175/2009JTECHA1332.1, 2010.

Baum, B. A., Yang, P., Heymsfield, A. J., Schmitt, C. G., Xie, Y., Bansemer, A., Hu, Y. X., and Zhang, Z.: Improvements in Shortwave Bulk Scattering and Absorption Models for the Remote Sensing of Ice Clouds, J. Appl. Meteorol. Clim., 50, 1037-1056, 2011.

Baumgardner, D., Jonsson, H., Dawson, W., O'Connor, D., and Newton, R.: The cloud, aerosol and precipitation spectrometer: a new instrument for cloud investigations, Atmos. Res., 59-60, 251-264, https://doi.org/10.1016/S0169-8095(01)001193, 2001.

Bohren, C. F. and Huffman, D. R.: Absorption and Scattering of Light by Small Particles, Wiley-Interscience, New York, 1983.
Fugal, J., Shaw, R., Saw, E., and Sergeyev, A.: Airborne digital holographic system for cloud particle measurements, Appl. Optics, 43, 5987-5995, 2004.

Garrett, T. J., Fallgatter, C., Shkurko, K., and Howlett, D.: Fall speed measurement and high-resolution multi-angle photography of hydrometeors in free fall, Atmos. Meas. Tech., 5, 26252633, https://doi.org/10.5194/amt-5-2625-2012, 2012.

Goodison, B. E., Louie, P. Y., and Yang, D.: WMO solid precipitation measurement intercomparison, World Meteorological Organization, Geneva, Switzerland, 1998.

Gultepe, I., Kuhn, T., Pavolonis, M., Calvert, C., Gurka, J., Heymsfield, A. J., Liu, P. S. K., Zhou, B., Ware, R., Ferrier, B., Milbrandt, J., and Berstein, B.: Ice Fog in Arctic During FRAM-Ice Fog Project: Aviation and Nowcasting Applications, B. Am. Meteorol. Soc., 95, 211-226, https://doi.org/10.1175/BAMS-D-11$00071.1,2014$.

Henneberger, J., Fugal, J. P., Stetzer, O., and Lohmann, U.: HOLIMO II: a digital holographic instrument for groundbased in situ observations of microphysical properties of mixed-phase clouds, Atmos. Meas. Tech., 6, 2975-2987, https://doi.org/10.5194/amt-6-2975-2013, 2013.

Kneifel, S., Löhnert, U., Battaglia, A., Crewell, S., and Siebler, D.: Snow scattering signals in ground-based passive microwave radiometer measurements, J. Geophys. Res., 115, D16214, https://doi.org/10.1029/2010JD013856, 2010.

Kruger, A. and Krajewski, W. F.: Two-dimensional video disdrometer: A brief description, J. Atmos. Ocean. Tech., 19, 602-617, https://doi.org/10.1175/15200426(2002)019<0602:TDVDAD>2.0.CO;2, 2002.

Kuhn, T.: Dual Ice Crystal Imager (D-ICI): images of snow particles, Kiruna, 2014, https://doi.org/10.5878/rhwc-7093, 2020.

Kuhn, T. and Gultepe, I.: Ice Fog and Light Snow Measurements Using a High-Resolution Camera System, Pure Appl. Geophys., 173, 3049-3064, https://doi.org/10.1007/s00024-0161343-7, 2016.

Kuhn, T., Grishin, I., and Sloan, J. J.: Improved Imaging and Image Analysis System for Application to Measurement of Small Ice Crystals, J. Atmos. Ocean. Tech., 29, 1811-1824, https://doi.org/10.1175/JTECH-D-11-00199.1, 2012.

Lawson, R. P., Baker, B. A., Schmitt, C. G., and Jensen, T. L.: An overview of microphysical properties of Arctic clouds observed in May and July 1998 during FIRE ACE, JGR, 106, 1498915014, 2001.

Loeb, N. G., Yang, P., Rose, F. G., Hong, G., Sun-Mack, S., Minnis, P., Kato, S., Ham, S.-H., Smith, W. L., Hioki, S., and Tang, G.: Impact of Ice Cloud Microphysics on Satellite Cloud Retrievals and Broadband Flux Radiative Transfer Model Calculations, J. Climate, 31, 1851-1864, https://doi.org/10.1175/JCLID-17-0426.1, 2018.

Magono, C. and Lee, C. W.: Meteorological Classification of Natural Snow Crystals, Journal of the Faculty of Science, Hokkaido University, Series 7, Geophysics, 2, 321-335, 1966.

Marchand, R., Mace, G. G., Hallar, A. G., McCubbin, I. B., Matrosov, S. Y., and Shupe, M. D.: Enhanced Radar Backscattering due to Oriented Ice Particles at $95 \mathrm{GHz}$ during StormVEx, J. Atmos. Ocean. Tech., 30, 2336-2351, https://doi.org/10.1175/JTECH-D-13-00005.1, 2013.

Matrosov, S. Y., Mace, G. G., Marchand, R., Shupe, M. D., Hallar, A. G., and McCubbin, I. B.: Observations of Ice Crystal Habits 
with a Scanning Polarimetric W-Band Radar at Slant Linear Depolarization Ratio Mode, J. Atmos. Ocean. Tech., 29, 989-1008, https://doi.org/10.1175/JTECH-D-11-00131.1, 2012.

McFarquhar, G. M. and Heymsfield, A. J.: Microphysical characteristics of three anvils sampled during the Central Equatorial Pacific Experiment, J. Atmos. Sci., 53, 2401-2423, 1996.

Mitchell, D. L.: Use of Mass and Area Dimensional Power Laws for Determining Precipitation Particle Terminal Velocities, J. Atmos. Sci., 53, 1710-1723, 1996.

Newman, A., Kucera, P., and Bliven, L.: Presenting the Snowflake Video Imager (SVI), J. Atmos. Ocean. Tech., 26, 167-179, https://doi.org/10.1175/2008JTECHA1148.1, 2009.

Schmitt, C. G., Stuefer, M., Heymsfield, A. J., and Kim, C. K.: The microphysical properties of ice fog measured in urban environments of Interior Alaska, J. Geophys. Res., 118, 11136-11147, https://doi.org/10.1002/jgrd.50822, 2013.

Schön, R., Schnaiter, M., Ulanowski, Z., Schmitt, C., Benz, S., Mohler, O., Vogt, S., Wagner, R., and Schurath, U.: Particle Habit Imaging Using Incoherent Light: A First Step toward a Novel Instrument for Cloud Microphysics, J. Atmos. Ocean. Tech., 28, 493-512, https://doi.org/10.1175/2011JTECHA1445.1, 2011.

Stoelinga, M. T., Hobbs, P. V., Mass, C. F., Locatelli, J. D., Colle, B. A., Houze, R. A., Rangno, A. L., and Bradley F. Smull, N. A. B., Rasmussen, R. M., Thompson, G., and Colman, B. R.: Improvement of Microphysical Parameterization through Observational Verification Experiment (IMPROVE), B. Am. Meteorol. Soc., 84, 1807-1826, https://doi.org/10.1175/bams-84-12-1807, 2003.
Sun, W., Hu, Y., Lin, B., Liu, Z., and Videen, G.: The impact of ice cloud particle microphysics on the uncertainty of ice water content retrievals, J. Quant. Spectrosc. Ra., 112, 189-196, https://doi.org/10.1016/j.jqsrt.2010.04.003, 2011.

Tao, W.-K., Simpson, J., Baker, D., Braun, S., Chou, M.-D., Ferrier, B., Johnson, D., Khain, A., Lang, S., Lynn, B., Shie, C.-L., Starr, D., Sui, C.-H., Wang, Y., and Wetzel, P.: Microphysics, radiation and surface processes in the Goddard Cumulus Ensemble (GCE) model, Met. Atm. Phys., 82, 97-137, 2003.

Vázquez-Martín, S., Kuhn, T., and Eliasson, S.: Shape dependence of falling snow crystals' microphysical properties using an updated shape classification, Appl. Sci., 10, 1163, https://doi.org/10.3390/app10031163, 2020.

Xie, Y., Yang, P., Kattawar, G. W., Baum, B. A., and Hu, Y.: Simulation of the optical properties of plate aggregates for application to the remote sensing of cirrus clouds, Appl. Optics, 50, 10651081, 2011.

Yang, P., Zhang, Z., Kattawar, G. W., Warren, S. G., Baum, B. A., Huang, H. L., Hu, Y. X., Winker, D., and Iaquinta, J.: Effect of cavities on the optical properties of bullet rosettes: Implications for active and passive remote sensing of ice cloud properties, $\mathrm{J}$ Appl. Meteorol. Clim., 47, 2311-330, 2008. 